\title{
ORTHOGONALLY ADDITIVE POLYNOMIALS ON THE ALGEBRAS OF APPROXIMABLE OPERATORS
}

\author{
J. ALAMINOS, M. L. C. GODOY, AND A. R. VILLENA
}

\begin{abstract}
Let $X$ and $Y$ be Banach spaces, let $\mathcal{A}(X)$ stands for the algebra of approximable operators on $X$, and let $P: \mathcal{A}(X) \rightarrow Y$ be an orthogonally additive, continuous $n$-homogeneous polynomial. If $X^{*}$ has the bounded approximation property, then we show that there exists a unique continuous linear map $\Phi: \mathcal{A}(X) \rightarrow Y$ such that $P(T)=\Phi\left(T^{n}\right)$ for each $T \in \mathcal{A}(X)$.
\end{abstract}

\section{INTRODUCTION}

Throughout all algebras and linear spaces are complex. Of course, linearity is understood to mean complex linearity.

Let $A$ be an algebra and let $Y$ be a linear space. A map $P: A \rightarrow Y$ is said to be orthogonally additive if

$$
a, b \in A, a b=b a=0 \Rightarrow P(a+b)=P(a)+P(b) .
$$

Let $X$ and $Y$ be linear spaces, and let $n \in \mathbb{N}$. A map $P: X \rightarrow Y$ is said to be an $n$-homogeneous polynomial if there exists an $n$-linear map $\varphi: X^{n} \rightarrow Y$ such that $P(x)=\varphi(x, \ldots, x)(x \in X)$. Here and subsequently, $X^{n}$ stands for the $n$-fold Cartesian product of $X$. Such a map is unique if it is required to be symmetric. This is a consequence of the so-called polarization formula which defines $\varphi$ through

$$
\varphi\left(x_{1}, \ldots, x_{n}\right)=\frac{1}{n ! 2^{n}} \sum_{\epsilon_{1}, \ldots, \epsilon_{n}= \pm 1} \epsilon_{1} \cdots \epsilon_{n} P\left(\epsilon_{1} x_{1}+\cdots+\epsilon_{n} x_{n}\right) .
$$

Further, in the case where $X$ and $Y$ are normed spaces, the polynomial $P$ is continuous if and only if the symmetric $n$-linear map $\varphi$ associated with $P$ is continuous.

Let $A$ be a Banach algebra. Given $n \in \mathbb{N}$, a Banach space $Y$, and a continuous linear map $\Phi: A \rightarrow Y$, the map $a \mapsto \Phi\left(a^{n}\right)$ is a typical example of continuous orthogonally additive $n$-homogeneous polynomial, and a standard problem consists in determining whether these are precisely the canonical examples of continuous orthogonally additive $n$-homogeneous polynomials on $A$. In the case where $A$ is a $C^{*}$-algebra, it is shown in [5] that every continuous $n$-homogeneous polynomial $P: A \rightarrow Y$ can be represented in the form

$$
P(a)=\Phi\left(a^{n}\right) \quad(a \in A)
$$

2010 Mathematics Subject Classification. 47H60, 46H35, 47L10.

Key words and phrases. Algebra of approximable operators; bounded approximation property; orthogonally additive polynomial.

The first and the third named authors were supported by MINECO grant MTM2015-65020-P and Junta de Andalucía grant FQM-185. The second named author was supported by Beca de iniciación a la investigación of Universidad de Granada. 
for some continuous linear map $\Phi: A \rightarrow Y$ (see [4, 6] for the case where $A$ is a $C^{*}$-algebra and $P$ is a holomorphic map). The references [1, 2, 7] discuss the case where $A$ is a commutative Banach algebra. This paper is concerned with the problem of representing the continuous orthogonally additive homogeneous polynomials in the case where $A$ is the algebra $\mathcal{A}(X)$ of approximable operators on a Banach space $X$. Here, $\mathcal{B}(X)$ is the Banach algebra of continuous linear operators on $X$, $\mathcal{F}(X)$ is the two-sided ideal of $\mathcal{B}(X)$ consisting of finite-rank operators, and $\mathcal{A}(X)$ is the closure of $\mathcal{F}(X)$ in $\mathcal{B}(X)$ with respect to the operator norm.

Let $X$ be a Banach space. Then $X^{*}$ denotes the dual of $X$. For $x \in X$ and $f \in X^{*}$, we write $x \otimes f$ for the operator defined by $(x \otimes f)(y)=f(y) x$ for each $y \in X$. Let $n \in \mathbb{N}$. Then we write $\mathbb{M}_{n}$ for the full matrix algebra of order $n$ over $\mathbb{C}$, and $\mathfrak{S}_{n}$ for the symmetric group of order $n$.

\section{Orthogonally additive Polynomials on the ALGEBRA OF Finite-RANK OPERATORS}

Lemma 2.1. Let $\mathcal{M}$ be a Banach algebra isomorphic to $\mathbb{M}_{k}$ for some $k \in \mathbb{N}$, let $Y$ be a Banach space, and let $P: \mathcal{M} \rightarrow Y$ be an orthogonally additive $n$-homogeneous polynomial. Then there exists a unique linear map $\Phi: \mathcal{M} \rightarrow Y$ such that

$$
P(a)=\Phi\left(a^{n}\right)
$$

for each $a \in \mathcal{M}$. Further, if $\varphi: \mathcal{M}^{n} \rightarrow Y$ is the symmetric n-linear map associated with $P$ and $e$ is the identity of $\mathcal{M}$, then

$$
\Phi(a)=\varphi(a, e, \ldots, e)
$$

for each $a \in \mathcal{M}$.

Proof. Let $\Psi: \mathcal{M} \rightarrow \mathbb{M}_{k}$ be an isomorphism. Since $\mathbb{M}_{k}$ is a $C^{*}$-algebra and the map $P \circ \Psi^{-1}: \mathbb{M}_{k} \rightarrow Y$ is easily seen to be an orthogonally additive $n$-homogeneous polynomial, [5, Corollary 3.1] then shows that there exists a unique linear map $\Theta: \mathbb{M}_{k} \rightarrow Y$ such that $P\left(\Psi^{-1}(M)\right)=\Theta\left(M^{n}\right)$ for each $M \in \mathbb{M}_{k}$. It is a simple matter to check that the map $\Phi=\Theta \circ \Psi$ satisfies (1). Now the polarization of (1) yields

$$
\varphi\left(a_{1}, \ldots, a_{n}\right)=\frac{1}{n !} \Phi\left(\sum_{\sigma \in \mathfrak{S}_{n}} a_{\sigma(1)} \cdots a_{\sigma(n)}\right)
$$

for each $\left(a_{1}, \ldots, a_{n}\right) \in \mathcal{M}$, whence $\varphi(a, e, \ldots, e)=\Phi(a)$ for each $a \in \mathcal{M}$.

Lemma 2.2. Let $X$ be a Banach space and let $T_{1}, \ldots, T_{m} \in \mathcal{F}(X)$. Then there exists a subalgebra $\mathcal{M}$ of $\mathcal{F}(X)$ such that $T_{1}, \ldots, T_{m} \in \mathcal{M}$ and $\mathcal{M}$ is isomorphic to $\mathbb{M}_{k}$ for some $k \in \mathbb{N}$.

Proof. We can certainly assume that $T_{1}, \ldots, T_{m}$ are rank-one operators. Write $T_{j}=x_{j} \otimes f_{j}$ with $x_{j} \in X$ and $f_{j} \in X^{*}$ for each $j \in\{1, \ldots, m\}$.

We claim that there exist $y_{1}, \ldots, y_{k} \in X$ and $g_{1}, \ldots g_{k} \in X^{*}$ such that

$$
\begin{aligned}
x_{1}, \ldots, x_{m} & \in \operatorname{span}\left(\left\{y_{1}, \ldots, y_{k}\right\}\right), \\
f_{1}, \ldots, f_{m} & \in \operatorname{span}\left(\left\{g_{1}, \ldots, g_{k}\right\}\right),
\end{aligned}
$$

and

$$
g_{i}\left(y_{j}\right)=\delta_{i j} \quad(i, j \in\{1, \ldots, k\}) .
$$


Let $\left\{g_{1}, \ldots, g_{l}\right\}$ be a basis of the linear span of $\left\{f_{1}, \ldots, f_{m}\right\}$, and let $y_{1} \ldots, y_{l} \in X$ be such that $g_{i}\left(y_{j}\right)=\delta_{i j}(i, j \in\{1, \ldots, l\})$. Let $U$ be the linear span of $\left\{y_{1}, \ldots, y_{l}\right\}$ in $X$, let $Q: X \rightarrow X / U$ the quotient map, and let

$$
U^{\perp}=\left\{f \in X^{*}: f\left(y_{1}\right)=\cdots=f\left(y_{l}\right)=0\right\} .
$$

If $\left\{x_{1}, \ldots, x_{m}\right\} \subset\left\{y_{1}, \ldots, y_{l}\right\}$, then our claim follows. We now assume that $\left\{x_{1}, \ldots, x_{m}\right\} \not \subset\left\{y_{1}, \ldots, y_{l}\right\}$. Let $y_{l+1}, \ldots, y_{k} \in X$ be such that $\left\{Q\left(y_{l+1}\right), \ldots, Q\left(y_{k}\right)\right\}$ is a basis of the linear span of the set $\left\{Q\left(x_{1}\right), \ldots, Q\left(x_{m}\right)\right\}$ in $X / U$. Since the map $f \mapsto f \circ Q$ defines an isometric isomorphism from $(X / U)^{*}$ onto $U^{\perp}$, it follows that there exist $g_{l+1}, \ldots, g_{k} \in U^{\perp}$ such that $g_{i}\left(y_{j}\right)=\delta_{i j}$ for all $i, j \in\{l+1, \ldots, k\}$. It is a simple matter to check that the sets $\left\{y_{1}, \ldots, y_{k}\right\}$ and $\left\{g_{1}, \ldots, g_{k}\right\}$ satisfy the requirements (3) and (4).

Let $\mathcal{M}$ be the subalgebra of $\mathcal{F}(X)$ generated by the set $\left\{y_{i} \otimes g_{j}: i, j \in\{1, \ldots, k\}\right\}$. By (3), $T_{1}, \ldots, T_{m} \in \mathcal{M}$. From (4) we conclude that the algebra $\mathcal{M}$ is isomorphic to the full matrix algebra $\mathbb{M}_{n}$. Actually, the map $T \mapsto\left[g_{i}\left(T\left(y_{j}\right)\right)\right]_{i, j}$ defines an isomorphism from $\mathcal{M}$ onto $\mathbb{M}_{k}$ that takes the operator $y_{i} \otimes g_{j}$ into the standard matrix unit $E_{i, j}(i, j \in\{1, \ldots, k\})$.

From Lemmas 2.1 and 2.2 we see immediately that each orthogonally additive $n$-homogeneous polynomial $P$ on $\mathcal{F}(X)$ can be represented in the standard way on any finitely generated subalgebra of $\mathcal{F}(X)$. The issue is whether the pieces $\Phi_{\mathcal{M}}$ (where $\mathcal{M}$ ranges over the finitely generated subalgebras of $\mathcal{F}(X)$ ) fit together to give a linear map $\Phi$ representing the polynomial $P$ on the whole $\mathcal{F}(X)$.

Corollary 2.3. Let $X$ and $Y$ be Banach spaces, and let $P: \mathcal{F}(X) \rightarrow Y$ be an orthogonally additive $n$-homogeneous polynomial. Then there exists a unique linear map $\Phi: \mathcal{F}(X) \rightarrow Y$ such that $P(T)=\Phi\left(T^{n}\right)$ for each $T \in \mathcal{F}(X)$.

Proof. Let $T \in \mathcal{F}(X)$. Lemma 2.2 shows that there exists a subalgebra $\mathcal{M}$ of $\mathcal{F}(X)$ such that $T \in \mathcal{M}$ and $\mathcal{M}$ is isomorphic to $\mathbb{M}_{k}$ for some $k \in \mathbb{N}$. Then Lemma 2.1 yields a unique linear map $\Phi_{\mathcal{M}}: \mathcal{M} \rightarrow Y$ such that $P(S)=\Phi_{\mathcal{M}}\left(S^{n}\right)$ for each $S \in \mathcal{M}$. Then we set $\Phi(T)=\Phi_{\mathcal{M}}(T)$. We now show that $\Phi$ is well-defined. Assume that $\mathcal{M}_{1}$ and $\mathcal{M}_{2}$ are subalgebras of $\mathcal{F}(X)$ with the properties that $T \in \mathcal{M}_{j}$ and $\mathcal{M}_{j}$ is isomorphic to a full matrix algebra $(j=1,2)$. Then, according to Lemma2.2, there exists a subalgebra $\mathcal{N}$ of $\mathcal{F}(X)$ such that $\mathcal{M}_{1}, \mathcal{M}_{2} \subset \mathcal{N}$ and $\mathcal{N}$ is isomorphic to a full matrix algebra. The uniqueness of the represention asserted in Lemma 2.1 gives that $\Phi_{\mathcal{N}}$ equals $\Phi_{\mathcal{M}_{j}}$ when restricted to $\mathcal{M}_{j}$ for $j=1,2$. Accordingly, we have $\Phi_{\mathcal{M}_{1}}(T)=\Phi_{\mathcal{N}}(T)=\Phi_{\mathcal{M}_{2}}(T)$.

Let $S, T \in \mathcal{F}(X)$ and let $\alpha, \beta \in \mathbb{C}$. By Lemma 2.2 there exists a subalgebra $\mathcal{M}$ of $\mathcal{F}(X)$ such that $S, T \in \mathcal{M}$ and $\mathcal{M}$ is isomorphic to a full matrix algebra. Then

$$
\Phi(\alpha S+\beta T)=\Phi_{\mathcal{M}}(\alpha S+\beta T)=\alpha \Phi_{\mathcal{M}}(S)+\beta \Phi_{\mathcal{M}}(T)=\alpha \Phi(S)+\beta \Phi(T),
$$

and, since $T^{n} \in \mathcal{M}$,

$$
P(T)=\Phi_{\mathcal{M}}\left(T^{n}\right)=\Phi\left(T^{n}\right) .
$$

This shows that $\Phi$ is linear and gives a representation of $P$. It should be pointed out that the polarization of this representations yields

$$
\varphi\left(T_{1}, \ldots, T_{n}\right)=\frac{1}{n !} \Phi\left(\sum_{\sigma \in \mathfrak{S}_{n}} T_{\sigma(1)} \cdots T_{\sigma(n)}\right)
$$


for each $\left(T_{1}, \ldots, T_{n}\right) \in \mathcal{F}(X)$, where $\varphi$ is the symmetric $n$-linear map associated with $P$.

Our final task is to prove the uniqueness of the map $\Phi$. Suppose that $\Psi: \mathcal{F}(X) \rightarrow$ $Y$ is a linear map such that $P(T)=\Psi\left(T^{n}\right)$ for each $T \in \mathcal{F}(X)$. The polarization of this identity gives

$$
\varphi\left(T_{1}, \ldots, T_{n}\right)=\frac{1}{n !} \Psi\left(\sum_{\sigma \in \mathfrak{S}_{n}} T_{\sigma(1)} \cdots T_{\sigma(n)}\right)
$$

for each $\left(T_{1}, \ldots, T_{n}\right) \in \mathcal{F}(X)$. Let $T \in \mathcal{F}(X)$. On account of Lemma 2.2, there exists $S \in \mathcal{F}(X)$ such that $T S=S T=T$. From (5) and (6) we obtain $\Phi(T)=$ $\varphi(T, S, \ldots, S)=\Psi(T)$.

It is not clear at all whether or not the linear map $\Phi$ given in the preceding result is continuous in the case where the polynomial $P$ is continuous.

\section{Orthogonally additive polynomials on the Algebra of APPROXIMABLE OPERATORS}

Let $A$ be a Banach algebra, let $\left(e_{\lambda}\right)_{\lambda \in \Lambda}$ be a bounded approximate identity for $A$ of bound $C$, and let $\mathcal{U}$ be an ultrafilter on $\Lambda$ containing the order filter on $\Lambda$ (which will be associated with $\left(e_{\lambda}\right)_{\lambda \in \Lambda}$ and fixed throughout). Let $Y$ be a dual Banach space and let $Y_{*}$ be a predual of $Y$. It follows from the Banach-Alaoglu theorem that each bounded subset of $Y$ is relatively compact with respect to the $\sigma\left(Y, Y_{*}\right)$ topology on $Y$. Consequently, each bounded net $\left(y_{\lambda}\right)_{\lambda \in \Lambda}$ in $Y$ has a unique limit with respect to the $\sigma\left(Y, Y_{*}\right)$-topology along the ultrafilter $\mathcal{U}$, and we write $\lim _{\mathcal{U}} y_{\lambda}$ for this limit.

Let $\varphi: A^{n} \rightarrow Y$ be a continuous $n$-linear map. For each $a_{1}, \ldots, a_{n-1} \in A$ and $\lambda \in \Lambda$, we have

$$
\begin{aligned}
\left\|\varphi\left(a_{1}, \ldots, a_{n-1}, e_{\lambda}\right)\right\| & \leq\|\varphi\|\left\|a_{1}\right\| \cdots\left\|a_{n-1}\right\|\left\|e_{\lambda}\right\| \\
& \leq C\|\varphi\|\left\|a_{1}\right\| \cdots\left\|a_{n-1}\right\| .
\end{aligned}
$$

Hence the net $\left(\varphi\left(a_{1}, \ldots, a_{n-1}, e_{\lambda}\right)\right)_{\lambda \in \Lambda}$ is bounded and therefore we can define the $\operatorname{map} \varphi^{\prime}: A^{n-1} \rightarrow Y$ by

$$
\varphi^{\prime}\left(a_{1}, \ldots, a_{n-1}\right)=\lim _{\mathcal{U}} \varphi\left(a_{1}, \ldots, a_{n-1}, e_{\lambda}\right)
$$

for each $\left(a_{1}, \ldots, a_{n-1}\right) \in A^{n-1}$. The linearity of the limit along an ultrafilter on a topological linear space gives the $(n-1)$-linearity of $\varphi^{\prime}$. Moreover, from (7) we deduce that

$$
\left\|\varphi^{\prime}\left(a_{1}, \ldots, a_{n-1}\right)\right\| \leq C\|\varphi\|\left\|a_{1}\right\| \cdots\left\|a_{n-1}\right\|
$$

for each $\left(a_{1}, \ldots, a_{n-1}\right) \in A^{n-1}$, which gives the continuity of $\varphi^{\prime}$ and $\left\|\varphi^{\prime}\right\| \leq C\|\varphi\|$. Further, it is clear that if the map $\varphi$ is symmetric, then the map $\varphi^{\prime}$ is symmetric.

Lemma 3.1. Let $A$ be a Banach algebra with a bounded approximate identity $\left(e_{\lambda}\right)_{\lambda \in \Lambda}$, let $Y$ be a dual Banach space, and let $\varphi: A^{n} \rightarrow Y$ be a continuous symmetric $n$-linear map with $n \geq 2$. Suppose that

$$
\varphi\left(a_{1}, \ldots, a_{n}\right)=\frac{1}{n !} \sum_{\sigma \in \mathfrak{S}_{n}} \varphi^{\prime}\left(a_{\sigma(1)}, \ldots, a_{\sigma(n-1)} a_{\sigma(n)}\right)
$$


for each $\left(a_{1}, \ldots, a_{n}\right) \in A^{n}$. Then there exists a continuous linear map $\Phi: A \rightarrow Y$ such that

$$
\varphi\left(a_{1}, \ldots, a_{n}\right)=\frac{1}{n !} \Phi\left(\sum_{\sigma \in \mathfrak{S}_{n}} a_{\sigma(1)} \cdots a_{\sigma(n)}\right)
$$

for each $\left(a_{1}, \ldots, a_{n}\right) \in A^{n}$.

Proof. The proof is by induction on $n$. The result is certainly true if $n=2$. Assume that the result is true for $n$, and let $\varphi: A^{n+1} \rightarrow Y$ be a continuous symmetric $(n+1)$-linear map such that

$$
\varphi\left(a_{1}, \ldots, a_{n}, a_{n+1}\right)=\frac{1}{(n+1) !} \sum_{\sigma \in \mathfrak{S}_{n+1}} \varphi^{\prime}\left(a_{\sigma(1)}, \ldots, a_{\sigma(n-1)}, a_{\sigma(n)} a_{\sigma(n+1)}\right)
$$

for each $\left(a_{1}, \ldots, a_{n+1}\right) \in A^{n+1}$. We claim that

$$
\varphi^{\prime}\left(a_{1}, \ldots, a_{n}\right)=\frac{1}{n !} \sum_{\sigma \in \mathfrak{S}_{n}} \varphi^{\prime \prime}\left(a_{\sigma(1)}, \ldots, a_{\sigma(n-1)} a_{\sigma(n)}\right)
$$

for each $\left(a_{1}, \ldots, a_{n}\right) \in A^{n}$. Here $\varphi^{\prime \prime}$ stands for the $(n-1)$-linear map $\left(\varphi^{\prime}\right)^{\prime}$. Indeed, for all $\left(a_{1}, \ldots, a_{n}\right) \in A^{n}$ and $\lambda \in \Lambda$, we have

$$
\begin{aligned}
\varphi\left(a_{1}, \ldots, a_{n}, e_{\lambda}\right)= & \frac{1}{(n+1) !} \sum_{\tau \in \mathfrak{S}_{n}} \varphi^{\prime}\left(a_{\tau(1)}, \ldots, a_{\tau(n-1)}, a_{\tau(n)} e_{\lambda}\right) \\
& +\frac{1}{(n+1) !} \sum_{\tau \in \mathfrak{S}_{n}} \varphi^{\prime}\left(a_{\tau(1)}, \ldots, a_{\tau(n-1)}, e_{\lambda} a_{\tau(n)}\right) \\
& +\frac{1}{(n+1) !} \sum_{\tau \in \mathfrak{S}_{n}} \varphi^{\prime}\left(a_{\tau(1)}, \ldots, e_{\lambda}, a_{\tau(n-1)} a_{\tau(n)}\right) \\
& +\cdots+\frac{1}{(n+1) !} \sum_{\tau \in \mathfrak{S}_{n}} \varphi^{\prime}\left(e_{\lambda}, a_{\tau(1)}, \ldots, a_{\tau(n-1)} a_{\tau(n)}\right) .
\end{aligned}
$$

Since $\left(e_{\lambda}\right)_{\lambda \in \Lambda}$ is a bounded approximate identity for $A$, the nets $\left(a_{k} e_{\lambda}\right)_{\lambda \in \Lambda}$ and $\left(e_{\lambda} a_{k}\right)_{\lambda \in \Lambda}$ converge to $a_{k}$ in norm for each $k \in\{1, \ldots, n\}$, and so, taking limits along $\mathcal{U}$ on both sides of the above equation (and using the continuity of $\varphi^{\prime}$ ), we see that

$$
\begin{aligned}
\varphi^{\prime}\left(a_{1}, \ldots, a_{n}\right)= & \lim _{\mathcal{U}} \varphi\left(a_{1}, \ldots, a_{n}, e_{\lambda}\right) \\
= & \frac{1}{(n+1) !} \sum_{\tau \in \mathfrak{S}_{n}} \lim _{\mathcal{U}} \varphi^{\prime}\left(a_{\tau(1)}, \ldots, a_{\tau(n-1)}, a_{\tau(n)} e_{\lambda}\right) \\
& +\frac{1}{(n+1) !} \sum_{\tau \in \mathfrak{S}_{n}} \lim _{\mathcal{U}} \varphi^{\prime}\left(a_{\tau(1)}, \ldots, a_{\tau(n-1)}, e_{\lambda} a_{\tau(n)}\right) \\
& +\frac{1}{(n+1) !} \sum_{\tau \in \mathfrak{S}_{n}} \lim _{\mathcal{U}} \varphi^{\prime}\left(a_{\tau(1)}, \ldots, e_{\lambda}, a_{\tau(n-1)} a_{\tau(n)}\right) \\
& +\cdots+\frac{1}{(n+1) !} \sum_{\tau \in \mathfrak{S}_{n}} \lim _{\mathcal{U}} \varphi^{\prime}\left(e_{\lambda}, a_{\tau(1)}, \ldots, a_{\tau(n-1)} a_{\tau(n)}\right) \\
= & \frac{1}{(n+1) !} \sum_{\tau \in \mathfrak{S}_{n}} \varphi^{\prime}\left(a_{\tau(1)}, \ldots, a_{\tau(n-1)}, a_{\tau(n)}\right)
\end{aligned}
$$




$$
\begin{aligned}
& +\frac{1}{(n+1) !} \sum_{\tau \in \mathfrak{S}_{n}} \varphi^{\prime}\left(a_{\tau(1)}, \ldots, a_{\tau(n-1)}, a_{\tau(n)}\right) \\
& +\frac{1}{(n+1) !} \sum_{\tau \in \mathfrak{S}_{n}} \varphi^{\prime \prime}\left(a_{\tau(1)}, \ldots, a_{\tau(n-1)} a_{\tau(n)}\right) \\
& +\cdots+\frac{1}{(n+1) !} \sum_{\tau \in \mathfrak{S}_{n}} \varphi^{\prime \prime}\left(a_{\tau(1)}, \ldots, a_{\tau(n-1)} a_{\tau(n)}\right) \\
& =\frac{1}{(n+1) !} 2 n ! \varphi^{\prime}\left(a_{1}, \ldots, a_{n-1}, a_{n}\right) \\
& +\frac{1}{(n+1) !}(n-1) \sum_{\tau \in \mathfrak{S}_{n}} \varphi^{\prime \prime}\left(a_{\tau(1)}, \ldots, a_{\tau(n-1)} a_{\tau(n)}\right) .
\end{aligned}
$$

We thus get

$$
\left(1-\frac{2}{n+1}\right) \varphi^{\prime}\left(a_{1}, \ldots, a_{n}\right)=\frac{n-1}{(n+1) !} \sum_{\tau \in \mathfrak{S}_{n}} \varphi^{\prime \prime}\left(a_{\tau(1)}, \ldots, a_{\tau(n-1)} a_{\tau(n)}\right),
$$

which proves our claim.

By (8) and the inductive hypothesis, there exists a continuous linear map $\Phi: A \rightarrow$ $Y$ such that

$$
\varphi^{\prime}\left(a_{1}, \ldots, a_{n}\right)=\frac{1}{n !} \Phi\left(\sum_{\tau \in \mathfrak{S}_{n}} a_{\tau(1)} \cdots a_{\tau(n)}\right)
$$

and thus

$$
\begin{aligned}
\varphi^{\prime}\left(a_{1}, \ldots, a_{n}\right)= & \frac{1}{n !} \sum_{\tau \in \mathfrak{S}_{n-1}} \Phi\left(a_{\tau(1)} \cdots a_{\tau(n-1)} a_{n}\right)+\frac{1}{n !} \sum_{\tau \in \mathfrak{S}_{n-1}} \Phi\left(a_{\tau(1)} \cdots a_{n} a_{\tau(n-1)}\right) \\
& +\cdots+\frac{1}{n !} \sum_{\tau \in \mathfrak{S}_{n-1}} \Phi\left(a_{n} a_{\tau(1)} \cdots a_{\tau(n-1)}\right)
\end{aligned}
$$

for each $\left(a_{1}, \ldots, a_{n}\right) \in A^{n}$. Therefore, for each $\left(a_{1}, \ldots, a_{n}, a_{n+1}\right) \in A^{n+1}$, we have

$$
\begin{aligned}
\varphi\left(a_{1}, \ldots, a_{n}, a_{n+1}\right)=\frac{1}{(n+1) !} \sum_{\sigma \in \mathfrak{S}_{n+1}} \varphi^{\prime}\left(a_{\sigma(1)}, \ldots, a_{\sigma(n-1)}, a_{\sigma(n)} a_{\sigma(n+1)}\right) \\
=\frac{1}{(n+1) !} \sum_{\sigma \in \mathfrak{S}_{n+1}} \frac{1}{n !} \sum_{\tau \in \mathfrak{S}_{n-1}} \Phi\left(a_{\sigma(\tau(1))} \cdots a_{\sigma(\tau(n-1))} a_{\sigma(n)} a_{\sigma(n+1)}\right) \\
+\frac{1}{(n+1) !} \sum_{\sigma \in \mathfrak{S}_{n+1}} \frac{1}{n !} \sum_{\tau \in \mathfrak{S}_{n-1}} \Phi\left(a_{\sigma(\tau(1))} \cdots a_{\sigma(n)} a_{\sigma(n+1)} a_{\sigma(\tau(n-1))}\right) \\
+\cdots+\frac{1}{(n+1) !} \sum_{\sigma \in \mathfrak{S}_{n+1}} \frac{1}{n !} \sum_{\tau \in \mathfrak{S}_{n-1}} \Phi\left(a_{\sigma(n)} a_{\sigma(n+1)} a_{\sigma(\tau(1))} \ldots a_{\sigma(\tau(n-1))}\right) \\
=\frac{1}{(n+1) !} \frac{1}{n !} \sum_{\tau \in \mathfrak{S}_{n-1}} \sum_{\sigma \in \mathfrak{S}_{n+1}} \Phi\left(a_{\sigma(\tau(1))} \cdots a_{\sigma(\tau(n-1))} a_{\sigma(n)} a_{\sigma(n+1)}\right) \\
+\frac{1}{(n+1) !} \frac{1}{n !} \sum_{\tau \in \mathfrak{S}_{n-1}} \sum_{\sigma \in \mathfrak{S}_{n+1}} \Phi\left(a_{\sigma(\tau(1))} \cdots a_{\sigma(n)} a_{\sigma(n+1)} a_{\sigma(\tau(n-1))}\right)
\end{aligned}
$$




$$
\begin{aligned}
& +\cdots+\frac{1}{(n+1) !} \frac{1}{n !} \sum_{\tau \in \mathfrak{S}_{n-1}} \sum_{\sigma \in \mathfrak{S}_{n+1}} \Phi\left(a_{\sigma(n)} a_{\sigma(n+1)} a_{\sigma(\tau(1))} \cdots a_{\sigma(\tau(n-1))}\right) \\
& =\frac{1}{(n+1) !} \frac{1}{n !} \sum_{\tau \in \mathfrak{S}_{n-1}} \sum_{\sigma \in \mathfrak{S}_{n+1}} \Phi\left(a_{\sigma(1)} \cdots a_{\sigma(n-1)} a_{\sigma(n)} a_{\sigma(n+1)}\right) \\
& +\frac{1}{(n+1) !} \frac{1}{n !} \sum_{\tau \in \mathfrak{S}_{n-1}} \sum_{\sigma \in \mathfrak{S}_{n+1}} \Phi\left(a_{\sigma(1)} \cdots a_{\sigma(n-1)} a_{\sigma(n)} a_{\sigma(n+1)}\right) \\
& +\cdots+\frac{1}{(n+1) !} \frac{1}{n !} \sum_{\tau \in \mathfrak{S}_{n-1}} \sum_{\sigma \in \mathfrak{S}_{n+1}} \Phi\left(a_{\sigma(1)} \cdots a_{\sigma(n-1)} a_{\sigma(n)} a_{\sigma(n+1)}\right) \\
& \quad=\frac{1}{(n+1) !} \sum_{\sigma \in \mathfrak{S}_{n+1}} \Phi\left(a_{\sigma(1)} \cdots a_{\sigma(n-1)} a_{\sigma(n)} a_{\sigma(n+1)}\right),
\end{aligned}
$$

and the induction continues.

Lemma 3.2. Let $\xi_{1}, \ldots, \xi_{n}, \zeta$ be noncommuting indeterminates and let $\pi_{n}$ be the polynomial defined by

$$
\pi_{n}\left(\xi_{1}, \ldots, \xi_{n}\right)=\sum_{\sigma \in \mathfrak{S}_{n}} \xi_{\sigma(1)} \cdots \xi_{\sigma(n)}
$$

Then the following identities hold:

$$
\begin{aligned}
& \sum_{\sigma \in \mathfrak{S}_{n}} \pi_{n}\left(\xi_{\sigma(1)}, \ldots, \xi_{\sigma(n)} \zeta\right)=(n-1) ! \sum_{\sigma \in \mathfrak{S}_{n}}\left[\xi_{\sigma(1)} \cdots \xi_{\sigma(n-1)} \xi_{\sigma(n)} \zeta\right. \\
&\left.+\xi_{\sigma(1)} \cdots \xi_{\sigma(n-1)} \zeta \xi_{\sigma(n)}+\cdots+\xi_{\sigma(1)} \zeta \cdots \xi_{\sigma(n-1)} \xi_{\sigma(n)}\right] \\
& \sum_{\sigma \in \mathfrak{S}_{n}} \pi_{n}\left(\xi_{\sigma(1)}, \ldots, \zeta \xi_{\sigma(n)}\right)=(n-1) ! \sum_{\sigma \in \mathfrak{S}_{n}}\left[\xi_{\sigma(1)} \cdots \xi_{\sigma(n-1)} \zeta \xi_{\sigma(n)}\right. \\
&\left.+\xi_{\sigma(1)} \cdots \zeta \xi_{\sigma(n-1)} \xi_{\sigma(n)}+\cdots+\zeta \xi_{\sigma(1)} \cdots \xi_{\sigma(n-1)} \xi_{\sigma(n)}\right]
\end{aligned}
$$

and

$$
\begin{gathered}
\sum_{\sigma \in \mathfrak{S}_{n}} \pi_{n}\left(\xi_{\sigma(1)}, \ldots, \xi_{\sigma(n-1)} \xi_{\sigma(n)}, \zeta\right)=(n-1) ! \sum_{\sigma \in \mathfrak{S}_{n}}\left[\xi_{\sigma(1)} \cdots \xi_{\sigma(n-1)} \xi_{\sigma(n)} \zeta\right. \\
\left.+\zeta \xi_{\sigma(1)} \cdots \xi_{\sigma(n-1)} \xi_{\sigma(n)}\right] \\
+(n-2)(n-2) ! \sum_{\sigma \in \mathfrak{S}_{n}}\left[\xi_{\sigma(1)} \cdots \xi_{\sigma(n-1)} \zeta \xi_{\sigma(n)}+\cdots+\xi_{\sigma(1)} \zeta \cdots \xi_{\sigma(n-1)} \xi_{\sigma(n)}\right] .
\end{gathered}
$$

Proof. It is clear that

$$
\pi_{n}\left(\xi_{1}, \ldots, \xi_{n}\right)=\sum_{\tau \in \mathfrak{S}_{n-1}}\left[\xi_{\tau(1)} \cdots \xi_{\tau(n-1)} \xi_{n}+\cdots+\xi_{n} \xi_{\tau(1)} \cdots \xi_{\tau(n-1)}\right] .
$$

Therefore, for each $\sigma \in \mathfrak{S}_{n}$, we have

$$
\begin{aligned}
\pi_{n}\left(\xi_{\sigma(1)}, \ldots, \xi_{\sigma(n)} \zeta\right)= & \sum_{\tau \in \mathfrak{S}_{n-1}}\left[\xi_{\sigma(\tau(1))} \cdots \xi_{\sigma(\tau(n-1))} \xi_{\sigma(n)} \zeta\right. \\
& \left.+\cdots+\xi_{\sigma(n)} \zeta \xi_{\sigma(\tau(1))} \cdots \xi_{\sigma(\tau(n-1))}\right]
\end{aligned}
$$


We thus get

$$
\begin{aligned}
& \sum_{\sigma \in \mathfrak{S}_{n}} \pi_{n}\left(\xi_{\sigma(1)}, \ldots, \xi_{\sigma(n)} \zeta\right) \\
& =\sum_{\tau \in \mathfrak{S}_{n-1}} \sum_{\sigma \in \mathfrak{S}_{n}}\left[\xi_{\sigma(\tau(1))} \cdots \xi_{\sigma(\tau(n-1))} \xi_{\sigma(n)} \zeta+\cdots+\xi_{\sigma(n)} \zeta \xi_{\sigma(\tau(1))} \cdots \xi_{\sigma(\tau(n-1))}\right] \\
& =\sum_{\tau \in \mathfrak{S}_{n-1}}\left[\sum_{\sigma \in \mathfrak{S}_{n}} \xi_{\sigma(1)} \cdots \xi_{\sigma(n-1)} \xi_{\sigma(n)} \zeta+\cdots+\sum_{\sigma \in \mathfrak{S}_{n}} \xi_{\sigma(1)} \zeta \cdots \xi_{\sigma(n-1)} \xi_{\sigma(n)}\right] \\
& \quad=(n-1) ! \sum_{\sigma \in \mathfrak{S}_{n}}\left[\xi_{\sigma(1)} \cdots \xi_{\sigma(n-1)} \xi_{\sigma(n)} \zeta+\cdots+\xi_{\sigma(1)} \zeta \cdots \xi_{\sigma(n-1)} \xi_{\sigma(n)}\right],
\end{aligned}
$$

which gives (9). In the same way we can check (10).

From (12) we now see that

$$
\begin{aligned}
\pi_{n}\left(\xi_{1}, \ldots, \xi_{n}\right)= & \sum_{\tau \in \mathfrak{S}_{n-2}}\left[\xi_{\tau(1)} \cdots \xi_{\tau(n-2)} \xi_{n-1} \xi_{n}+\xi_{\tau(1)} \cdots \xi_{n-1} \xi_{\tau(n-2)} \xi_{n}\right. \\
& \left.+\cdots+\xi_{n-1} \xi_{\tau(1)} \cdots \xi_{\tau(n-2)} \xi_{n}\right] \\
+ & \sum_{\tau \in \mathfrak{S}_{n-2}}\left[\xi_{\tau(1)} \cdots \xi_{\tau(n-2)} \xi_{n} \xi_{n-1}+\xi_{\tau(1)} \cdots \xi_{n-1} \xi_{n} \xi_{\tau(n-2)}\right. \\
& \left.+\cdots+\xi_{n-1} \xi_{\tau(1)} \cdots \xi_{n} \xi_{\tau(n-2)}\right] \\
+ & \sum_{\tau \in \mathfrak{S}_{n-2}}\left[\xi_{\tau(1)} \cdots \xi_{n} \xi_{\tau(n-2)} \xi_{n-1}+\xi_{\tau(1)} \cdots \xi_{n} \xi_{n-1} \xi_{\tau(n-2)}\right. \\
& \left.+\cdots+\xi_{n-1} \xi_{\tau(1)} \cdots \xi_{n} \xi_{\tau(n-3)} \xi_{\tau(n-2)}\right] \\
+ & \cdots+ \\
+ & \sum_{\tau \in \mathfrak{S}_{n-2}}\left[\xi_{n} \xi_{\tau(1)} \cdots \xi_{\tau(n-2)} \xi_{n-1}+\xi_{n} \xi_{\tau(1)} \cdots \xi_{n-1} \xi_{\tau(n-2)}\right. \\
& \left.+\cdots+\xi_{n} \xi_{n-1} \xi_{\tau(1)} \cdots \xi_{\tau(n-3)} \xi_{\tau(n-2)}\right] .
\end{aligned}
$$

Therefore, for each $\sigma \in \mathfrak{S}_{n}$, we have

$$
\begin{aligned}
& \pi_{n}\left(\xi_{\sigma(1)}, \ldots, \xi_{\sigma(n-1)} \xi_{\sigma(n)}, \zeta\right) \\
& =\sum_{\tau \in \mathfrak{S}_{n-2}}\left[\xi_{\sigma(\tau(1))} \cdots \xi_{\sigma(\tau(n-2))} \xi_{\sigma(n-1)} \xi_{\sigma(n)} \zeta+\xi_{\sigma(\tau(1))} \cdots \xi_{\sigma(n-1)} \xi_{\sigma(n)} \xi_{\sigma(\tau(n-2))} \zeta\right. \\
& \left.\quad+\cdots+\xi_{\sigma(n-1)} \xi_{\sigma(n)} \xi_{\sigma(\tau(1))} \cdots \xi_{\sigma(\tau(n-2))} \zeta\right] \\
& \quad+\sum_{\tau \in \mathfrak{S}_{n-2}}\left[\xi_{\sigma(\tau(1))} \cdots \xi_{\sigma(\tau(n-2))} \zeta \xi_{\sigma(n-1)} \xi_{\sigma(n)}+\xi_{\sigma(\tau(1))} \cdots \xi_{\sigma(n-1)} \xi_{\sigma(n)} \zeta \xi_{\sigma(\tau(n-2))}\right. \\
& \left.\quad+\cdots+\xi_{\sigma(n-1)} \xi_{\sigma(n)} \xi_{\sigma(\tau(1))} \cdots \zeta \xi_{\sigma(\tau(n-2))}\right] \\
& \quad+\sum_{\tau \in \mathfrak{S}_{n-2}}\left[\xi_{\sigma(\tau(1))} \cdots \zeta \xi_{\sigma(\tau(n-2))} \xi_{\sigma(n-1)} \xi_{\sigma(n)}+\xi_{\sigma(\tau(1))} \cdots \zeta \xi_{\sigma(n-1)} \xi_{\sigma(n)} \xi_{\sigma(\tau(n-2))}\right.
\end{aligned}
$$




$$
\begin{aligned}
& \left.\quad+\cdots+\xi_{\sigma(n-1)} \xi_{\sigma(n)} \xi_{\sigma(\tau(1))} \cdots \zeta \xi_{\sigma(\tau(n-3))} \xi_{\sigma(\tau(n-2))}\right] \\
& +\cdots+ \\
& +\sum_{\tau \in \mathfrak{S}_{n-2}}\left[\zeta \xi_{\sigma(\tau(1))} \cdots \xi_{\sigma(\tau(n-2))} \xi_{\sigma(n-1)} \xi_{\sigma(n)}+\zeta \xi_{\sigma(\tau(1))} \cdots \xi_{\sigma(n-1)} \xi_{\sigma(n)} \xi_{\sigma(\tau(n-2))}\right. \\
& \left.\quad+\cdots+\zeta \xi_{\sigma(n-1)} \xi_{\sigma(n)} \xi_{\sigma(\tau(1))} \cdots \xi_{\sigma(\tau(n-3))} \xi_{\sigma(\tau(n-2))}\right] .
\end{aligned}
$$

We thus get

$$
\begin{aligned}
& \sum_{\sigma \in \mathfrak{S}_{n}} \pi_{n}\left(\xi_{\sigma(1)}, \ldots, \xi_{\sigma(n-1)} \xi_{\sigma(n)}, \zeta\right) \\
& =\sum_{\tau \in \mathfrak{S}_{n-2}}\left[\sum_{\sigma \in \mathfrak{S}_{n}} \xi_{\sigma(\tau(1))} \cdots \xi_{\sigma(\tau(n-2))} \xi_{\sigma(n-1)} \xi_{\sigma(n)} \zeta\right. \\
& +\sum_{\sigma \in \mathfrak{S}_{n}} \xi_{\sigma(\tau(1))} \cdots \xi_{\sigma(n-1)} \xi_{\sigma(n)} \xi_{\sigma(\tau(n-2))} \zeta \\
& \left.+\cdots+\sum_{\sigma \in \mathfrak{S}_{n}} \xi_{\sigma(n-1)} \xi_{\sigma(n)} \xi_{\sigma(\tau(1))} \cdots \xi_{\sigma(\tau(n-2))} \zeta\right] \\
& +\sum_{\tau \in \mathfrak{S}_{n-2}}\left[\sum_{\sigma \in \mathfrak{S}_{n}} \xi_{\sigma(\tau(1))} \cdots \xi_{\sigma(\tau(n-2))} \zeta \xi_{\sigma(n-1)} \xi_{\sigma(n)}\right. \\
& +\sum_{\sigma \in \mathfrak{S}_{n}} \xi_{\sigma(\tau(1))} \cdots \xi_{\sigma(n-1)} \xi_{\sigma(n)} \zeta \xi_{\sigma(\tau(n-2))} \\
& \left.+\cdots+\sum_{\sigma \in \mathfrak{S}_{n}} \xi_{\sigma(n-1)} \xi_{\sigma(n)} \xi_{\sigma(\tau(1))} \cdots \zeta \xi_{\sigma(\tau(n-2))}\right] \\
& +\sum_{\tau \in \mathfrak{S}_{n-2}}\left[\sum_{\sigma \in \mathfrak{S}_{n}} \xi_{\sigma(\tau(1))} \cdots \zeta \xi_{\sigma(\tau(n-2))} \xi_{\sigma(n-1)} \xi_{\sigma(n)}\right. \\
& +\sum_{\sigma \in \mathfrak{S}_{n}} \xi_{\sigma(\tau(1))} \cdots \zeta \xi_{\sigma(n-1)} \xi_{\sigma(n)} \xi_{\sigma(\tau(n-2))} \\
& \left.+\cdots+\sum_{\sigma \in \mathfrak{S}_{n}} \xi_{\sigma(n-1)} \xi_{\sigma(n)} \xi_{\sigma(\tau(1))} \cdots \zeta \xi_{\sigma(\tau(n-3))} \xi_{\sigma(\tau(n-2))}\right] \\
& +\cdots+ \\
& +\sum_{\tau \in \mathfrak{S}_{n-2}}\left[\sum_{\sigma \in \mathfrak{S}_{n}} \zeta \xi_{\sigma(\tau(1))} \cdots \xi_{\sigma(\tau(n-2))} \xi_{\sigma(n-1)} \xi_{\sigma(n)}\right. \\
& +\sum_{\sigma \in \mathfrak{S}_{n}} \zeta \xi_{\sigma(\tau(1))} \cdots \xi_{\sigma(n-1)} \xi_{\sigma(n)} \xi_{\sigma(\tau(n-2))} \\
& \left.+\cdots+\sum_{\sigma \in \mathfrak{S}_{n}} \zeta \xi_{\sigma(n-1)} \xi_{\sigma(n)} \xi_{\sigma(\tau(1))} \cdots \xi_{\sigma(\tau(n-3))} \xi_{\sigma(\tau(n-2))}\right] \\
& =\sum_{\tau \in \mathfrak{S}_{n-2}}\left[\sum_{\sigma \in \mathfrak{S}_{n}} \xi_{\sigma(1)} \cdots \xi_{\sigma(n-2)} \xi_{\sigma(n-1)} \xi_{\sigma(n)} \zeta+\sum_{\sigma \in \mathfrak{S}_{n}} \xi_{\sigma(1)} \cdots \xi_{\sigma(n-2)} \xi_{\sigma(n-1)} \xi_{\sigma(n)} \zeta\right. \\
& \left.+\cdots+\sum_{\sigma \in \mathfrak{S}_{n}} \xi_{\sigma(1)} \xi_{\sigma(2)} \xi_{\sigma(3)} \cdots \xi_{\sigma(n)} \zeta\right]
\end{aligned}
$$




$$
\begin{aligned}
& +\sum_{\tau \in \mathfrak{S}_{n-2}}\left[\sum_{\sigma \in \mathfrak{S}_{n}} \xi_{\sigma(1)} \cdots \xi_{\sigma(n-2)} \zeta \xi_{\sigma(n-1)} \xi_{\sigma(n)}+\sum_{\sigma \in \mathfrak{S}_{n}} \xi_{\sigma(1)} \cdots \xi_{\sigma(n-2)} \xi_{\sigma(n-1)} \zeta \xi_{\sigma(n)}\right. \\
& \left.+\cdots+\sum_{\sigma \in \mathfrak{S}_{n}} \xi_{\sigma(1)} \xi_{\sigma(2)} \xi_{\sigma(3)} \cdots \zeta \xi_{\sigma(n)}\right] \\
& +\sum_{\tau \in \mathfrak{S}_{n-2}}\left[\sum_{\sigma \in \mathfrak{S}_{n}} \xi_{\sigma(1)} \cdots \zeta \xi_{\sigma(n-2)} \xi_{\sigma(n-1)} \xi_{\sigma(n)}+\sum_{\sigma \in \mathfrak{S}_{n}} \xi_{\sigma(1)} \cdots \zeta \xi_{\sigma(n-2)} \xi_{\sigma(n-1)} \xi_{\sigma(n)}\right. \\
& \left.+\cdots+\sum_{\sigma \in \mathfrak{S}_{n}} \xi_{\sigma(1)} \xi_{\sigma(2)} \xi_{\sigma(3)} \cdots \zeta \xi_{\sigma(n-1)} \xi_{\sigma(n)}\right] \\
& +\cdots+ \\
& +\sum_{\tau \in \mathfrak{S}_{n-2}}\left[\sum_{\sigma \in \mathfrak{S}_{n}} \zeta \xi_{\sigma(1)} \cdots \xi_{\sigma(n-2)} \xi_{\sigma(n-1)} \xi_{\sigma(n)}+\sum_{\sigma \in \mathfrak{S}_{n}} \zeta \xi_{\sigma(1)} \cdots \xi_{\sigma(n-2)} \xi_{\sigma(n-1)} \xi_{\sigma(n)}\right. \\
& \left.+\cdots+\sum_{\sigma \in \mathfrak{S}_{n}} \zeta \xi_{\sigma(1)} \xi_{\sigma(2)} \xi_{\sigma(3)} \cdots \xi_{\sigma(n-1)} \xi_{\sigma(n)}\right] \\
& =\sum_{\tau \in \mathfrak{S}_{n-2}} \sum_{\sigma \in \mathfrak{S}_{n}}\left[(n-1) \xi_{\sigma(1)} \cdots \xi_{\sigma(n-2)} \xi_{\sigma(n-1)} \xi_{\sigma(n)} \zeta\right] \\
& +\sum_{\tau \in \mathfrak{S}_{n-2}} \sum_{\sigma \in \mathfrak{S}_{n}}\left[\xi_{\sigma(1)} \cdots \xi_{\sigma(n-2)} \zeta \xi_{\sigma(n-1)} \xi_{\sigma(n)}+(n-2) \xi_{\sigma(1)} \cdots \xi_{\sigma(n-2)} \xi_{\sigma(n-1)} \zeta \xi_{\sigma(n)}\right] \\
& +\sum_{\tau \in \mathfrak{S}_{n-2}} \sum_{\sigma \in \mathfrak{S}_{n}}\left[2 \xi_{\sigma(1)} \cdots \zeta \xi_{\sigma(n-2)} \xi_{\sigma(n-1)} \xi_{\sigma(n)}+(n-3) \xi_{\sigma(1)} \cdots \xi_{\sigma(n-2)} \zeta \xi_{\sigma(n-1)} \xi_{\sigma(n)}\right] \\
& +\cdots+ \\
& +\sum_{\tau \in \mathfrak{S}_{n-2}} \sum_{\sigma \in \mathfrak{S}_{n}}\left[k \xi_{\sigma(1)} \cdots \zeta \xi_{\sigma(n-k)} \cdots \xi_{\sigma(n)}+(n-k-1) \xi_{\sigma(1)} \cdots \xi_{\sigma(n-k)} \zeta \cdots \xi_{\sigma(n)}\right] \\
& +\cdots+ \\
& +\sum_{\tau \in \mathfrak{S}_{n-2}} \sum_{\sigma \in \mathfrak{S}_{n}}\left[(n-1) \zeta \xi_{\sigma(1)} \cdots \xi_{\sigma(n-2)} \xi_{\sigma(n-1)} \xi_{\sigma(n)}\right] \\
& =(n-2) ! \sum_{\sigma \in \mathfrak{S}_{n}}\left[(n-1) \xi_{\sigma(1)} \cdots \xi_{\sigma(n-2)} \xi_{\sigma(n-1)} \xi_{\sigma(n)} \zeta\right] \\
& +(n-2) ! \sum_{\sigma \in \mathfrak{S}_{n}}\left[\xi_{\sigma(1)} \cdots \xi_{\sigma(n-2)} \zeta \xi_{\sigma(n-1)} \xi_{\sigma(n)}+(n-2) \xi_{\sigma(1)} \cdots \xi_{\sigma(n-2)} \xi_{\sigma(n-1)} \zeta \xi_{\sigma(n)}\right] \\
& +(n-2) ! \sum_{\sigma \in \mathfrak{S}_{n}}\left[2 \xi_{\sigma(1)} \cdots \zeta \xi_{\sigma(n-2)} \xi_{\sigma(n-1)} \xi_{\sigma(n)}+(n-3) \xi_{\sigma(1)} \cdots \xi_{\sigma(n-2)} \zeta \xi_{\sigma(n-1)} \xi_{\sigma(n)}\right] \\
& +\cdots+ \\
& +(n-2) ! \sum_{\sigma \in \mathfrak{S}_{n}}\left[k \xi_{\sigma(1)} \cdots \zeta \xi_{\sigma(n-k)} \cdots \xi_{\sigma(n)}+(n-k-1) \xi_{\sigma(1)} \cdots \xi_{\sigma(n-k)} \zeta \cdots \xi_{\sigma(n)}\right] \\
& +\cdots+ \\
& +(n-2) ! \sum_{\sigma \in \mathfrak{S}_{n}}\left[(n-1) \zeta \xi_{\sigma(1)} \cdots \xi_{\sigma(n-2)} \xi_{\sigma(n-1)} \xi_{\sigma(n)}\right] \text {, }
\end{aligned}
$$

which gives (11). 
Theorem 3.3. Let $X$ and $Y$ be Banach spaces, and let $P: \mathcal{A}(X) \rightarrow Y$ be a continuous n-homogeneous polynomial. Suppose that $X^{*}$ has the bounded approximation property. Then the following conditions are equivalent:

(1) the polynomial $P$ is orthogonally additive, i.e., $P(S+T)=P(S)+P(T)$ whenever $S, T \in \mathcal{A}(X)$ are such that $S T=T S=0$;

(2) the polynomial $P$ is orthogonally additive on $\mathcal{F}(X)$, i.e., $P(S+T)=P(S)+$ $P(T)$ whenever $S, T \in \mathcal{F}(X)$ are such that $S T=T S=0$;

(3) there exists a unique continuous linear map $\Phi: \mathcal{A}(X) \rightarrow Y$ such that $P(T)=$ $\Phi\left(T^{n}\right)$ for each $T \in \mathcal{A}(X)$.

Proof. It is clear that $(1) \Rightarrow(2)$ and that $(3) \Rightarrow(1)$. We will henceforth prove that $(2) \Rightarrow(3)$.

Since $P$ is orthogonally additive on $\mathcal{F}(X)$, Corollary 2.3 yields a linear map $\Phi_{0}: \mathcal{F}(X) \rightarrow Y$ such that

$$
P(T)=\Phi_{0}\left(T^{n}\right) \quad(T \in \mathcal{F}(X)) .
$$

It seems to be appropriate to emphasize that we don't know whether or not $\Phi_{0}$ is continuous, despite the continuity of $P$.

Let $\varphi: \mathcal{A}(X)^{n} \rightarrow Y$ be the continuous symmetric $n$-linear map associated with $P$. By considering the natural embedding of $Y$ into the second dual $Y^{* *}$ we regard $Y$ as a closed linear subspace of $Y^{* *}$, and we regard $\varphi$ as a continuous $n$-linear map from $\mathcal{A}(X)^{n}$ into $Y^{* *}$ henceforth. Since $X^{*}$ has the bounded approximation property, it follows that $\mathcal{A}(X)$ has a bounded approximate identity $\left(E_{\lambda}\right)_{\lambda \in \Lambda}$ ([3, Theorem 2.9.37(iii) and (v)]) and we may assume that $\left(E_{\lambda}\right)_{\lambda \in \Lambda}$ lies in $\mathcal{F}(X)$. Let $\varphi^{\prime}: \mathcal{A}(X)^{n-1} \rightarrow Y^{* *}$ be as defined in the beginning of this section. The polarization of the identity (13) yields

$$
\varphi\left(T_{1}, \ldots, T_{n}\right)=\frac{1}{n !} \Phi_{0}\left(\pi_{n}\left(T_{1}, \ldots, T_{n}\right)\right) \quad\left(\left(T_{1}, \ldots, T_{n}\right) \in \mathcal{F}(X)^{n}\right)
$$

where $\pi_{n}$ is the noncommutative polynomial introduced in Lemma 3.2 .

We claim that

$$
\varphi\left(T_{1}, \ldots, T_{n}\right)=\frac{1}{n !} \sum_{\sigma \in \mathfrak{S}_{n}} \varphi^{\prime}\left(T_{\sigma(1)}, \ldots, T_{\sigma(n-1)} T_{\sigma(n)}\right)
$$

for each $\left(T_{1}, \ldots, T_{n}\right) \in \mathcal{A}(X)^{n}$. Since $\mathcal{A}(X)$ is the closed linear span of the rank-one operators and both $\varphi$ and $\varphi^{\prime}$ are continuous, we are reduced to proving (15) in the case where $T_{1}, \ldots, T_{n}$ are rank-one operators. Let $T_{1}, \ldots, T_{n} \in \mathcal{F}(X)$ be rank-one operators, and let $\lambda \in \Lambda$. On account of (9), (10), (11), and (14), we have

$$
\begin{aligned}
& \sum_{\sigma \in \mathfrak{S}_{n}} \varphi\left(T_{\sigma(1)}, \ldots, T_{\sigma(n)} E_{\lambda}\right) \\
= & \frac{1}{n} \Phi_{0}\left(\sum_{\sigma \in \mathfrak{S}_{n}}\left[T_{\sigma(1)} \cdots T_{\sigma(n-1)} T_{\sigma(n)} E_{\lambda}+\cdots+T_{\sigma(1)} E_{\lambda} \cdots T_{\sigma(n-1)} T_{\sigma(n)}\right]\right), \\
& \sum_{\sigma \in \mathfrak{S}_{n}} \varphi\left(T_{\sigma(1)}, \ldots, E_{\lambda} T_{\sigma(n)}\right) \\
= & \frac{1}{n} \Phi_{0}\left(\sum_{\sigma \in \mathfrak{S}_{n}}\left[T_{\sigma(1)} \cdots T_{\sigma(n-1)} E_{\lambda} T_{\sigma(n)}+\cdots+E_{\lambda} T_{\sigma(1)} \cdots T_{\sigma(n-1)} T_{\sigma(n)}\right]\right),
\end{aligned}
$$




$$
\begin{aligned}
& \sum_{\sigma \in \mathfrak{S}_{n}} \varphi\left(T_{\sigma(1)}, \ldots, T_{\sigma(n-1)} T_{\sigma(n)}, E_{\lambda}\right) \\
&= \frac{1}{n} \Phi_{0}\left(\sum_{\sigma \in \mathfrak{S}_{n}}\left[T_{\sigma(1)} \cdots T_{\sigma(n-1)} T_{\sigma(n)} E_{\lambda}+E_{\lambda} T_{\sigma(1)} \cdots T_{\sigma(n-1)} T_{\sigma(n)}\right]\right) \\
&+\frac{n-2}{n(n-1)} \Phi_{0}\left(\sum_{\sigma \in \mathfrak{S}_{n}}\left[T_{\sigma(1)} \cdots T_{\sigma(n-1)} E_{\lambda} T_{\sigma(n)}+\cdots+T_{\sigma(1)} E_{\lambda} \cdots T_{\sigma(n-1)} T_{\sigma(n)}\right]\right) .
\end{aligned}
$$

Adding (16) and (17), and then subtracting (18) we can assert that

$$
\begin{gathered}
\sum_{\sigma \in \mathfrak{S}_{n}} \varphi\left(T_{\sigma(1)}, \ldots, T_{\sigma(n)} E_{\lambda}\right)+\sum_{\sigma \in \mathfrak{S}_{n}} \varphi\left(T_{\sigma(1)}, \ldots, E_{\lambda} T_{\sigma(n)}\right) \\
-\sum_{\sigma \in \mathfrak{S}_{n}} \varphi\left(T_{\sigma(1)}, \ldots, T_{\sigma(n-1)} T_{\sigma(n)}, E_{\lambda}\right) \\
=\frac{1}{n-1} \Phi_{0}\left(\sum_{\sigma \in \mathfrak{S}_{n}}\left[T_{\sigma(1)} \cdots T_{\sigma(n-1)} E_{\lambda} T_{\sigma(n)}+\cdots+T_{\sigma(1)} E_{\lambda} \cdots T_{\sigma(n-1)} T_{\sigma(n)}\right]\right) .
\end{gathered}
$$

Let $\mathcal{M}$ be the subalgebra of $\mathcal{F}(X)$ generated by $T_{1}, \ldots, T_{n}$. Then $\mathcal{M}$ is finitedimensional, a property which implies that the restriction of $\Phi_{0}$ to $\mathcal{M}$ is continuous, of course. Further, it is straightforward to check that $T_{i} S T_{j} \in \mathcal{M}$ for all $S \in \mathcal{B}(X)$ and $i, j \in\{1, \ldots, n\}$. Accordingly, for each $\sigma \in \mathfrak{S}_{n}$, all the nets

$$
\left(T_{\sigma(1)} \cdots T_{\sigma(n-1)} E_{\lambda} T_{\sigma(n)}\right)_{\lambda \in \Lambda}, \ldots,\left(T_{\sigma(1)} E_{\lambda} \cdots T_{\sigma(n-1)} T_{\sigma(n)}\right)_{\lambda \in \Lambda}
$$

lie in $\mathcal{M}$, and, since $\left(E_{\lambda}\right)_{\lambda \in \Lambda}$ is a bounded approximate identity for $\mathcal{A}(X)$, each of them converges to $T_{\sigma(1)} \cdots T_{\sigma(n-1)} T_{\sigma(n)}$ in the operator norm. Therefore, taking limits along $\mathcal{U}$ on both sides of the equation (19) (and using the continuity of $\Phi_{0}$ on $\mathcal{M})$, we see that

$$
\begin{gathered}
\sum_{\sigma \in \mathfrak{S}_{n}} \varphi\left(T_{\sigma(1)}, \ldots, T_{\sigma(n)}\right)+\sum_{\sigma \in \mathfrak{S}_{n}} \varphi\left(T_{\sigma(1)}, \ldots, T_{\sigma(n)}\right)-\sum_{\sigma \in \mathfrak{S}_{n}} \varphi^{\prime}\left(T_{\sigma(1)}, \ldots, T_{\sigma(n-1)} T_{\sigma(n)}\right) \\
=\frac{1}{n-1} \Phi_{0}\left(\sum_{\sigma \in \mathfrak{S}_{n}}\left[T_{\sigma(1)} \cdots T_{\sigma(n-1)} T_{\sigma(n)}+\cdots+T_{\sigma(1)} \cdots T_{\sigma(n-1)} T_{\sigma(n)}\right]\right) \\
=\Phi_{0}\left(\sum_{\sigma \in \mathfrak{S}_{n}} T_{\sigma(1)} \cdots T_{\sigma(n-1)} T_{\sigma(n)}\right) .
\end{gathered}
$$

By using the symmetry of $\varphi$ and (14) in (20) we obtain

$$
2 n ! \varphi\left(T_{1}, \ldots, T_{n}\right)-\sum_{\sigma \in \mathfrak{S}_{n}} \varphi^{\prime}\left(T_{\sigma(1)}, \ldots, T_{\sigma(n-1)} T_{\sigma(n)}\right)=n ! \varphi\left(T_{1}, \ldots, T_{n}\right),
$$

which yields (15).

Having disposed of identity (15), we can now apply Lemma 3.1 to obtain a continuous linear map $\Phi: \mathcal{A}(X) \rightarrow Y^{* *}$ such that

$$
\varphi\left(T_{1}, \ldots, T_{n}\right)=\frac{1}{n !} \Phi\left(\sum_{\sigma \in \mathfrak{S}_{n}} T_{\sigma(1)} \cdots T_{\sigma(n)}\right)
$$


for each $\left(T_{1}, \ldots, T_{n}\right) \in \mathcal{A}(X)$. Our next objective is to show that the range of $\Phi$ lies actually in $Y$. Let $T \in \mathcal{F}(X)$. On account of Lemma 2.2, there exists $S \in \mathcal{F}(X)$ such that $T S=S T=T$. From (21) we see that $\Phi(T)=\varphi(T, S, \ldots, S) \in Y$. Since $\mathcal{F}(X)$ is dense in $\mathcal{A}(X), \Phi$ is continuous, and $Y$ is closed in $Y^{* *}$, it may be concluded that $\Phi(\mathcal{A}(X)) \subset Y$.

Our final task is to prove the uniqueness of the map $\Phi$. Suppose that $\Psi: \mathcal{A}(X) \rightarrow$ $Y$ is a continuous linear map such that $P(T)=\Psi\left(T^{n}\right)$ for each $T \in \mathcal{A}(X)$. By Corollary 2.3, $\Psi(T)=\Phi(T)\left(=\Phi_{0}(T)\right)$ for each $T \in \mathcal{F}(X)$. Since $\mathcal{F}(X)$ is dense in $\mathcal{A}(X)$, and both $\Phi$ and $\Psi$ are continuous, it follows that $\Psi(T)=\Phi(T)$ for each $T \in \mathcal{A}(X)$.

Remark 1. Let $X$ and $Y$ be as in Theorem 3.3, let $P: \mathcal{A}(X) \rightarrow Y$ be a continuous orthogonally additive $n$-homogeneous polynomial, and let $\varphi: \mathcal{A}(X)^{n} \rightarrow Y$ be the continuous symmetric $n$-linear map associated with $P$. Suppose that $\left(E_{\lambda}\right)_{\lambda \in \Lambda}$ is any bounded approximate identity for $\mathcal{A}(X)$. We already know that there exists a continuous linear map $\Phi: \mathcal{A}(X) \rightarrow Y$ such that $P(T)=\Phi\left(T^{n}\right)$ for each $T \in \mathcal{A}(X)$. The polarization of this representation gives

$$
\varphi\left(T_{1}, \ldots, T_{n}\right)=\frac{1}{n !} \Phi\left(\sum_{\sigma \in \mathfrak{S}_{n}} T_{\sigma(1)} \cdots T_{\sigma(n)}\right)
$$

for each $\left(T_{1}, \ldots, T_{n}\right) \in \mathcal{A}(X)$. If $T \in \mathcal{A}(X)$, then each of the nets

$$
\left(T E_{\lambda} \cdots E_{\lambda}\right)_{\lambda \in \Lambda},\left(E_{\lambda} T \cdots E_{\lambda}\right)_{\lambda \in \Lambda}, \ldots,\left(E_{\lambda} \cdots T E_{\lambda}\right)_{\lambda \in \Lambda},\left(E_{\lambda} \cdots E_{\lambda} T\right)_{\lambda \in \Lambda},
$$

converges to $T$ in the operator norm, and using (22) and the continuity of $\Phi$ we see that the net $\left(\varphi\left(T, E_{\lambda}, \ldots, E_{\lambda}\right)\right)_{\lambda \in \Lambda}$ converges to $\Phi(T)$ in norm. Accordingly, the map $\Phi$ is necessarily given by

$$
\Phi(T)=\lim _{\lambda \in \Lambda} \varphi\left(T, E_{\lambda}, \ldots, E_{\lambda}\right) \quad(T \in \mathcal{A}(X)),
$$

where there is no need for taking the limit with respect to any weak topology along any ultrafilter. Note the similarity of (23) with (2).

\section{REFERENCES}

[1] J. Alaminos, M. Brešar, Š. Špenko, A. R. Villena. Orthogonally additive polynomials and orthosymmetric maps in Banach algebras with properties $\mathbb{A}$ and $\mathbb{B}$. Proc. Edinb. Math. Soc. 59 (3) (2016) 559-568.

[2] J. Alaminos, J. Extremera, A. R. Villena. Orthogonally additive polynomials on Fourier algebras. J. Math. Anal. Appl. 422 (2015) 72-83.

[3] H.G. Dales, Banach algebras and automatic continuity, London Mathematical Society Monographs, New Series, 24, Oxford Science Publications, The Clarendon Press, Oxford University Press, New York, 2000.

[4] J. J. Garcés, A. M. Peralta, D. Puglisi, M. I. Ramírez. Orthogonally additive and orthogonality preserving holomorphic mappings between $C^{*}$-algebras. Abstr. Appl. Anal. 2013 (2013) Art. ID 415354.

[5] C. Palazuelos, A. M. Peralta, I. Villanueva. Orthogonally additive polynomials on $C^{*}$ algebras. Q. J. Math. 59 (2008), 363-374.

[6] A. M. Peralta, D. Puglisi. Orthogonally additive holomorphic functions on $C^{*}$-algebras. Oper. Matrices 6 (2012) 621-629.

[7] A. R. Villena. Orthogonally additive polynomials on Banach function algebras. J. Math. Anal. Appl. 448 (2017) 447-472. 
Departamento de Análisis Matemático, Facultad de Ciencias, Universidad de Granada, 18071 Granada, Spain

E-mail address: alaminos@ugr.es

Departamento de Análisis Matemático, Facultad de Ciencias, Universidad de Granada, 18071 Granada, Spain

E-mail address: mlcg.godoy@gmail.com

Departamento de Análisis Matemático, Facultad de Ciencias, Universidad de Granada, 18071 Granada, Spain

E-mail address: avillena@ugr.es 